\title{
The Effect of Nanofilled Resin-Base Coating on the Mechanical and Physical Properties of Resin Composites
}

\author{
Negar Moghaddasi ${ }^{1}$ Maryam Tavallali ${ }^{1}$ Dana Jafarpour ${ }^{1} \quad$ Reihaneh Ferooz $^{2}$ Rafat Bagheri ${ }^{3}$
}

\author{
${ }^{1}$ Department of Physiotherapy, Biomaterials Research Centre, Shiraz \\ University of Medical Sciences, Shiraz, Iran \\ ${ }^{2}$ Masters of Musculoskeletal Physiotherapy Candidate, La Trobe \\ University, Melbourne, Australia \\ ${ }^{3}$ Department of Dental Materials, Biomaterials Research Centre, School \\ of Dentistry, Shiraz University of Medical Sciences, Shiraz, Iran
}

\author{
Address for correspondence Rafat Bagheri, DDS, PhD, \\ Department of Dental Materials, School of Dentistry, \\ Shiraz University of Medical Sciences, Shiraz 43111, Iran \\ (e-mail: bagherir@unimelb.edu.au).
}

Eur J Dent 2021;15:202-209

\begin{abstract}
Keywords

- coating

- resin composite

- water sorption/ solubility

- color

- microhardness

- flexural strength

Objective The aim of this study was to evaluate the effects of a nanofilled resin-base coating on the physical and mechanical properties of four different types of resin composites.

Materials and Methods Disc-shaped specimens of four resin composites (Aura bulk fil [SDI], IPS Impress Direct [Ivoclar], Filtek Z250, and Filtek P60 [3M/ESPE]) were prepared and divided in two groups: either uncoated or coated with G-coat plus $(n=10)$. The specimens were obtained and stored in distilled water (DW) at $37^{\circ} \mathrm{C}$. For water sorption and solubility, the specimens were stored for 60 days in DW and measured according to ISO 4049 instructions. The specimens were subjected to color change $(\Delta \mathrm{E})$, microhardness, and flexural strength (FS) measurements after 24 hours and 60 days of immersion in DW.

Statistical Analysis ANOVA, post hoc Tukey's, and independent $t$-tests were used to analyze data.

Results Mean solubility values of coated groups were significantly lower than that of uncoated ones $(p<0.001)$. No significant differences were detected between neither water sorption nor FS of coated and uncoated groups $(p=0.502$ and $p=0.510$, respectively). For all materials except IPS Empress, the mean values for $\Delta \mathrm{E}$ were not statistically different between coated and uncoated groups, and mean microhardness values of the uncoated groups were significantly greater than the coated groups $(p<0.001)$. Conclusion Coating resin composites improved water sorption and solubility but increased $\Delta \mathrm{E}$, decreased microhardness, and had no significant effect on FS in the long run.
\end{abstract}

\section{Introduction}

The physical and mechanical properties of restorative materials are the main consideration in selecting them for dental practice. An ideal restorative material needs to be strong enough to endure the mastication forces, and resist against

published online October 27, 2020
DOI https://doi.org/ $10.1055 / \mathrm{s}-0040-1716784$ ISSN 1305-7456. degradation and discoloration in the harsh environment of oral cavity. Resin composites are highly sought for esthetic restorations because of its various range of shades and compatibility with enamel. ${ }^{1}$ The most important advantages of resin composite is its ability to bond to enamel micromechanically, ${ }^{2}$ and its longevity which has been reported for

\section{(c) 2020. European Journal of Dentistry.}

This is an open access article published by Thieme under the terms of the Creative Commons Attribution-NonDerivative-NonCommercial-License, permitting copying and reproduction so long as the original work is given appropriate credit. Contents may not be used for commercial purposes, or adapted, remixed, transformed or built upon. (https://creativecommons.org/licenses/by-nc-nd/4.0/)

Thieme Medical and Scientific Publishers Pvt. Ltd., A-12, 2nd Floor, Sector 2, Noida-201301 UP, India 
approximately 7 years. ${ }^{3}$ Hardness, flexural strength (FS), color stability, and water sorption/solubility are the critical and relevant mechanical and physical properties to be investigated.

Previous studies have shown that the mechanical properties of resin-based restorative materials will be significantly influenced by aging and water storage due to sorption and solubility. ${ }^{4,5}$ Sorption arising from the polymer matrix hydrolytically degrades the network structure, de-bonds the silanized filler, and consequently influences the solubility of these materials. ${ }^{6}$ Moreover, the effect of storage in distilled water (DW) on the hardness of esthetic restorative materials is well established. ${ }^{7.8}$ Discoloration of resin restorative materials is also affected by a variety of factors including sorption/ solubility and the material's composition. ${ }^{9-11}$

To prevent the surface of esthetic restorative materials from moisture contamination, improving their durability in the moist oral environment, a coating agent was introduced. ${ }^{12} \mathrm{G}$-coat Plus is a self-adhesive nanofill resin base coating that provides maximum surface protection and contains single phase dispersed nanofillers ( $30 \mathrm{~nm}$ ) that is light polymerized. ${ }^{13}$ Several studies found that protective resin coating may reduce microleakage, ${ }^{14}$ and increase color stability and FS of resin-based materials. ${ }^{12,15}$ Hankins et al studied the influence of a nanofill resin-based coating application on water absorption by teeth filled with glass ionomer cement, concluding that resin coating may reduce water absorption. ${ }^{16}$ Others have reported a positive effect of G-Coat Plus on the shear punch and FS of resin-based restorative materials, yet a negative effect on their hardness. ${ }^{12}$

Although A positive effect of resin-coating on the mechanical and physical properties of RMGICs has been reported in previous studies, ${ }^{12,15,17,18}$ the influence of resin coating on the physical and mechanical properties of different types of resin composites and their possible long-term effects remain unknown.

To simulate the clinical efficacy of nanofill resin-base coating, our study aimed to examine and compare the mechanical (Vickers microhardness and FS) and physical (sorption/ solubility and color stability) properties of Aura, IPS Empress Direct, Filtek Z250, and Filtek P60 with and without applying G-Coat Plus up to 60 days. The null hypothesis was that the application of G-Coat Plus would not change the physical or mechanical properties of those resin composites.

\section{Materials and Methods}

The materials used in this study are presented in - Table $\mathbf{1}$.

\section{Specimen Preparation for Physical Properties}

For sorption/solubility and color stability tests, 40 disc-shaped specimens $(10.0 \mathrm{~mm}$ diameter $\times 1.0 \mathrm{~mm}$ thick) were prepared for each material. The material was positioned in the appropriate plastic mold and hand-pressed between two transparent plastic matrix strips and glass plates to obtain a flat surface. Materials were light cured through the transparent matrix in accordance with the manufactures' instruction for 20 seconds by means of a LED curing unit (Radii plus LED; SDI, Victoria, Australia) with a wavelength of 440 to $480 \mathrm{~mm}$ and emitting light intensity of $1,500 \mathrm{~mW} / \mathrm{cm}^{2}$. Then the specimens were detached from the mold and manually wet polished in the edges by using 1,000 grit silicon carbide paper. The disc-shaped specimens of each material were subdivided randomly into two groups: 20 coated and 20 uncoated. G-Coat Plus was applied to both sides of the specimens in the coated group by using a brush and then light cured based on the manufacturer's suggested exposure time. Half of the specimens in each group were exposed to sorption/ solubility tests $(n=10)$, and half were subjected to color measurements $(n=10)$. Measurements of sorption/solubility and color stability were conducted as below.

\section{Water Sorption/Solubility}

According to ISO 4049's instructions, the specimens were put in a desiccator (Labx Company; Ontario, Canada) holding freshly dried silica gel (Sigma-Aldrich; Taufkirchen, Germany) at $37^{\circ} \mathrm{C}$ for 22 hours and then at $25^{\circ} \mathrm{C}$ for 2 hours. The weight of the specimens was measured after 24 hours on a digital

Table 1 Description of all the resin composites used in the study

\begin{tabular}{|c|c|c|c|c|c|}
\hline $\begin{array}{l}\text { Resin } \\
\text { composite }\end{array}$ & Type & Manufacturer & Resin matrix & $\begin{array}{l}\text { Filler content (wt\%), } \\
\text { type, size }\end{array}$ & Lot number \\
\hline aura (Bulkfill) & Nano-Hybrid & $\begin{array}{l}\text { SDI, Melbourne, } \\
\text { Australia }\end{array}$ & $\begin{array}{l}\text { UDMA, Bis-EMA, Bis-GMA, } \\
\text { TEGDMA }\end{array}$ & $\begin{array}{l}\text { Amorphous } \mathrm{SiO}_{2} \text {, Barium } \\
\text { aluminosilicate glass, } \\
\text { prepolymerized filler (72.71\%) }\end{array}$ & 160841 \\
\hline IPS Empress & Nano-Hybrid & $\begin{array}{l}\text { Ivoclar } \\
\text { Vivadent AG, } \\
\text { Liechtenstein }\end{array}$ & Bis-GMA, TEGDMA, DMAEMA & $\begin{array}{l}\text { Barium-aluminum-fluorosilicate } \\
\text { glass, ytterbium trifluoride, } \\
\text { prepolymerized filler (79\%) }\end{array}$ & V10919 \\
\hline Filtek Z250 & Micro-Hybrid & 3M ESPE, USA & $\begin{array}{l}\text { Bis-GMA, UDMA, bis-EMA, } \\
\text { TEGDMA }\end{array}$ & Zirconia, Silica (78\%) & N694978 \\
\hline Filtek P60 & Micro-Hybrid & 3M ESPE, USA & Bis-GMA, UDMA, bis-EMA & $\begin{array}{l}\text { Zirconia, Silica }(0.01-3.5) \mu \mathrm{m}, \\
(61 \%)\end{array}$ & N511095 \\
\hline G-Coat Plus & $\begin{array}{l}\text { Light-cured } \\
\text { resin coating }\end{array}$ & GC Japan & $\begin{array}{l}\text { Urethane methacrylate/methyl } \\
\text { methacrylate/camphorquinone/ } \\
\text { silicon dioxide/phosphoric ester } \\
\text { monomer }\end{array}$ & & 1311121 \\
\hline
\end{tabular}

Abbreviations: Bis-GMA, bisphenol A-glycidyl methacrylate; Bis-EMA, ethoxylatedbisphenol-A-dimethacrylate; DMAEMA, dimethyl amino ethyl methacrylate; TEGDMA, triethylene glycol dimethacrylate; UDMA, urethane dimethacrylate. 
scale (GR-3000; A \& D CL Toshiba, Tokyo, Japan) to an accurateness of $\pm 0.1 \mathrm{mg}$. This procedure was performed each day until a constant weight was attained $\left(\mathrm{M}_{0}\right)$. Then the specimens were immersed into DW for 60 days, removed, blotted dry, and weighed again until the mass became constant $\left(\mathrm{M}_{1}\right)$. The water was changed weekly in the first 60 days. Then the specimens were put back in the desiccator for another 60 days and recorded $\left(\mathrm{M}_{2}\right)$, all masses are in micrograms. Thickness and diameter of each specimen were measured, calculating the volume $(V): V=\pi \times r^{2} \times h$. Using the following equations, the water sorption (Wsp) and solubility $\left(\mathrm{Ws}_{1}\right)$ values were calculated in $\mu \mathrm{g} / \mathrm{mm}^{3}$ : $\mathrm{Ws}_{1}=\left(\mathrm{m}_{0}-\mathrm{m}_{1}\right) / \mathrm{V}$, Wsp $=\left(\mathrm{m}_{1}-\mathrm{m}_{2}\right) / \mathrm{V}$

\section{Color Change}

The color of specimens was measured after 24 hours and 60 days of immersion in DW in the dark room. The specimens were removed from DW, blotted dry with tissue paper, and placed on a white background. The color of the specimens was assessed using the Commission Internationale d'Eclairge $\mathrm{L}^{*} \mathrm{a}^{*} \mathrm{~b}^{*}$ color coordination (CIE L*a*b*). The color alteration value $(\triangle \mathrm{E})$ between 24 hours and 60 days of measurement was measured with the following formula: $\Delta \mathrm{E}=\left[(\Delta \mathrm{L})^{2}+(\Delta \mathrm{a})^{2}+(\Delta \mathrm{b})^{2}\right]^{1 / 2}$.

\section{Specimen Preparation for Mechanical Properties}

For FS and Vickers hardness number (VHN) tests, a total of 40 bar-shaped specimens of $2 \mathrm{~mm}$ in height, $5 \mathrm{~mm}$ in width, and $25 \mathrm{~mm}$ in length were prepared for each material. Restorative materials were located in the mold between two matrix strip and glass slabs, and were pressed to extrude the spare material. The materials were cured in three equal sections according to manufacturer recommended time for 20 seconds on each section. This procedure was repeated for the opposite side after the specimen were removed from the mold. Specimen boundaries were wet polished manually using a silicon carbide paper in a circular motion. Specimens were randomly divided into two groups: coated $(n=20)$ and uncoated $(n=20)$. A thin layer of GCP was applied on one surface of the coated group using a brush and cured by means of the light curing unit for 20 seconds. Then specimens of each group were subdivided into another two groups and kept in DW at $37^{\circ} \mathrm{C}$ for either 24 hours $(n=10)$ or 60 days $(n=10)$. Following each time interval, the test specimens were removed from the water, and FS and VHN were measured as below.

\section{Flexural Strength Test}

Prior to the test, the width and height of each specimen was measured using a digital caliper (Absolute Caliper; Mitutoyo Kawasaki, Japan) with a precision of $0.1 \mathrm{~mm}$. The specimens were positioned in the universal testing machine (ZwickRoell Group; Germany) by means of a 4-point bend test jig. The coated layer was faced down in a way that maximum tensile loads were inserted on this surface. The specimens were loaded at a crosshead speed of $0.5 \mathrm{~mm} /$ minute. The maximum stress at failure was documented, and FS was measured with the following formula: $r=3 . F .\left(L_{1}-L_{2}\right) / 2 . b . d,{ }^{2}$ where $F$ is the force at the fracture point $(\mathrm{N}) ; \mathrm{L}_{1}$ and $\mathrm{L}_{2}$ are the outer and inner roll span of the jig respectively; $b$ is specimens width $(\mathrm{mm})$; and $\mathrm{d}$ is the specimens height $(\mathrm{mm})$.

\section{Vickers Microhardness Number}

The specimen's halves remained from the fracture toughness test were to test hardness. Three specimens were selected from each group and subjected to three indentations $35 \mu \mathrm{m}$ apart across the specimen surface by means of a Vickers hardness tester (MHV-1000Z; SCTMC, Shanghai, China) $(n=3 \times 3$ indentation $=9$ ). A $300 \mathrm{~g}$ load was applied for 10 seconds. The average microhardness number was recorded as VHN.

\section{Data Analysis}

Data were described using mean and standard deviation $( \pm S D)$. Kolmogorov-Smirnov test was employed for assessing normality assumption. Two-way ANOVA was applied to investigate any interaction between materials and coatings for the water sorption and solubility tests, and three-way ANOVA was employed to analyze the effect of the factors (material, coating, and time) on the response variables for Vickers microhardness and FS tests. In the case of a significant interaction effect, subgroup analyzes using one-way ANOVA/Tukey honestly significant difference and independent $t$-tests were performed. The significant level was set to be $\alpha=0.05$.

\section{Results}

\section{Comparison of Water Sorption and Solubility}

The normality assumption was confirmed in all cases. - Table 2 shows means \pm SD for water sorption (Wsp) and solubility (Wsl) variables. Two-way ANOVA for Wsp variable indicated that there was no significant material $\times$ condition interaction effect $(p=0.998)$. The results of two-way ANOVA are also shown in - Table 2 for both variables. Main effect analyzes revealed no significant difference between coated (5.24 \pm 1.66 ) and uncoated ( $5.48 \pm 1.52$ ) groups by controlling the type of material $(p=0.502)$. However, there was a significant difference between mean Wsp of the four materials $(p<0.001)$ when the effect of condition had been controlled. Mean Wsp of Aura (3.32 \pm 1.00$)$ was significantly lower than other materials $(p<0.001)$, followed by IPS Empress $(5.41 \pm$ $0.96)$ and Filtek Z250 (6.07 $\pm 1.11 ; p=0.001)$. There was no significant difference between Filtek Z250 and Filtek P60 (6.66 $\pm 0.73 ; p=0.209)$.

No significant interaction effect was found for Wsl $(p=0.999)$. By controlling the effect of material type, mean Wsl of coated groups were significantly lower than that of uncoated groups $(p<0.001)$. There was also a significant difference between mean Wsl of the four materials $(p<0.001)$ by controlling the effect of condition. Mean Wsl of both Aura and IPS Empress were greater than those of Filtek Z250 (-2.03 \pm 3.56$)$ and Filtek P60 (-3.62 \pm 0.78 ; all $p<0.001)$.

\section{Comparison of Color Change}

There was a significant interaction effect between material and condition $(p=0.008)$. Therefore, comparisons were made based on subgroup analyzes instead of main effect analyzes. The mean $\Delta \mathrm{E}$ values are presented in - Table 3. Coated and uncoated groups were not statistically different for all 
Table 2 Mean \pm standard deviation of water sorption and solubility $\left(\mu \mathrm{g} / \mathrm{mm}^{3}\right)$

\begin{tabular}{|c|c|c|c|c|c|c|}
\hline \multirow[t]{2}{*}{ Variable } & \multirow[t]{2}{*}{ Material } & \multicolumn{2}{|c|}{ Condition } & \multirow[t]{2}{*}{ Total } & \multicolumn{2}{|c|}{ Two-way ANOVA results } \\
\hline & & Coated & Uncoated & & Effect & $p$-Value \\
\hline \multirow[t]{5}{*}{ Water sorption } & Aura & $3.28 \pm 1.12$ & $3.36 \pm 0.91$ & $3.32 \pm 1.00$ & & \\
\hline & IPS & $5.19 \pm 0.82$ & $5.36 \pm 1.07$ & $5.41 \pm 0.96$ & Material & $<0.001$ \\
\hline & FZ250 & $5.70 \pm 1.07$ & $6.37 \pm 1.10$ & $6.07 \pm 1.11$ & Condition & 0.502 \\
\hline & FP60 & $7.01 \pm 0.81$ & $6.34 \pm 0.50$ & $6.66 \pm 0.73$ & Interaction & 0.998 \\
\hline & Total & $5.24 \pm 1.66$ & $5.48 \pm 1.52$ & & & \\
\hline \multirow[t]{5}{*}{ Water solubility } & Aura & $-0.60 \pm 1.27$ & $2.45 \pm 2.20$ & $0.92 \pm 2.34$ & & \\
\hline & IPS & $-1.10 \pm 0.81$ & $1.15 \pm 1.30$ & $0.09 \pm 1.57$ & Material & $<0.001$ \\
\hline & $\mathrm{FZ250}$ & $-3.82 \pm 1.29$ & $-0.04 \pm 4.26$ & $-2.03 \pm 3.56$ & Condition & $<0.001$ \\
\hline & FP60 & $-3.36 \pm 0.75$ & $-3.85 \pm 0.76$ & $-3.62 \pm 0.78$ & Interaction & 0.999 \\
\hline & Total & $-2.22 \pm 1.76$ & $-0.08 \pm 3.37$ & & & \\
\hline
\end{tabular}

Note: For better illustration, the values were multiplied by 100,000 .

Table 3 Mean \pm standard deviation of color change values

\begin{tabular}{|l|l|l|l|l|}
\hline \multirow{2}{*}{ Material } & \multicolumn{2}{|c|}{ Condition } & \multicolumn{2}{|c|}{ Two-way ANOVA results } \\
\cline { 2 - 5 } & Coated & Uncoated & Effect & -Value \\
\hline Aura & $7.75 \pm 2.98^{\mathrm{a}, \mathrm{AB}}$ & $6.53 \pm 1.52^{\mathrm{a}, \mathrm{A}}$ & Material & 0.001 \\
IPS & $10.86 \pm 4.26^{\mathrm{a}, \mathrm{A}}$ & Condition & 0.013 \\
\cline { 1 - 2 } FZ250 & $7.31 \pm 3.86^{\mathrm{a}, \mathrm{AB}}$ & & 0.008 \\
\cline { 1 - 3 } & $5.37 \pm 2.95^{\mathrm{a}, \mathrm{B}}$ & $8.78 \pm 4.11^{\mathrm{b}, \mathrm{AB}}$ & \\
\end{tabular}

Note: In each condition, mean values with at least a common capital letter were not statistically different (one-way ANOVA/Tukey's post hoc test). In each row, mean values with at least a common small letter were not statistically different (independent $t$-test).

materials except for IPS Empress, the mean $\Delta \mathrm{E}$ of the coated group $(10.86 \pm 4.26)$ was greater than the uncoated one $(5.37 \pm$ $1.81 ; p=0.003)$. Among coated specimens, mean $\Delta \mathrm{E}$ of IPS Empress was significantly greater than only Filtek P60 (5.37 \pm $2.95 ; p=0.024)$. In uncoated groups, mean $\Delta \mathrm{E}$ of Filtek Z250 $(8.78 \pm 4.13)$ and Aura $(6.53 \pm 1.52)$ were significantly greater than Filtek P60 ( $3.47 \pm 1.96 ; p_{1}=0.008$ and $p_{2}=0.017$, respectively). In this case, the other groups were statistically similar.

\section{Comparison of Flexural Strength}

A three-way ANOVA was used to analyze the effect of material, condition, and time on FS. Mean FS for different combination of material, condition, and time are shown in - Table 4. There was only a significant interaction effect between material and time $(p=0.001)$. Therefore, main effect results were reported only for condition and subgroup analyzes approach was used to assess the effect of different combinations of material and time.

By controlling the effect of time and material, the overall mean FS of the coated groups (57.96 \pm 20.12$)$ and uncoated ones $(58.89 \pm 23.28)$ were not statistically different ( $p=0.510)$. After 24 hours, Filtek P60 had the greatest mean FS, followed by Aura $(p<0.05)$. After 60 days, the Filtek P60 revealed significantly higher FS than Aura (48.20 \pm 8.98$)$ and Filtek Z250 (48.87 \pm 17.93 ) with no significant differences between the other materials.
In Aura, time 1 (24 hours; $65.58 \pm 9.51$ ) provided a greater mean FS than time 2 (60 days; $48.20 \pm 8.98 ; p<0.001$ ). But, the mean FS of time $1(37.96 \pm 8.17)$ was lower than time 2 $(48.87 \pm 17.93)$ in IPS Empress $(p=0.044)$. In other material, the two time points were not statistically different in mean FS.

\section{Comparison of Vickers Microhardness}

The three-way analyzes of ANOVA for VHN showed that all interaction effects were significant. Therefore, subgroup analyzes were done to study the effects of the three variables. - Table 5 shows mean microhardness for different combination of subgroups. Mean VHN of the materials were significant in each combination of conditions and time points. In coated groups after 24 hours, the mean VHN of Filtek P60 was significantly greater than other materials (all $p<0.001$ ) and after 60 days, there was a significant difference only between Filtek Z250 (41.28 \pm 7.16) and Aura (30.28 \pm 5.03; $p=0.024)$. In uncoated groups after 24 hours, Filtek P60 $(74.08 \pm 6.04)$ had the greatest mean VHN values when compared with the other materials $(p<0.001)$. Moreover, mean VHN of Filtek Z250 (44.80 \pm 3.97$)$ and Aura (41.14 \pm 1.94$)$ were significantly greater that IPS Empress $(26.27 \pm 0.74)$. In uncoated groups of 60 days, Filtek P60 (71.75 \pm 3.32$)$ and Filtek Z250 ( $70.24 \pm 5.83)$ had the significantly greatest mean VHN values $(p<0.001)$. In addition, Aura $(41.94 \pm 1.85)$ had a greater mean VHN than IPS Empress (33.55 $\pm 1.34 ; p<0.001)$. 
Table 4 Mean \pm standard deviation of flexural strength values

\begin{tabular}{|l|l|l|l|l|l|l|}
\hline \multirow{2}{*}{ Material } & \multicolumn{2}{|c|}{ Coated } & \multicolumn{2}{c|}{ Uncoated } & \multicolumn{2}{c|}{ Total } \\
\cline { 2 - 7 } & 24 hours & 60 days & 24 hours & 60 days & Time 1 & Time 2 \\
\hline Aura & $65.93 \pm 8.20$ & $48.86 \pm 9.563$ & $65.23 \pm 9.56$ & $47.54 \pm 8.96$ & $65.58 \pm 9.51^{\mathrm{a}, \mathrm{A}}$ & $48.20 \pm 8.98^{\mathrm{b}, \mathrm{A}}$ \\
\hline IPS & $38.15 \pm 10.15$ & $52.99 \pm 17.07$ & $37.73 \pm 17.07$ & $44.75 \pm 18.95$ & $37.96 \pm 8.17^{\mathrm{a}, \mathrm{B}}$ & $44.75 \pm 18.95^{\mathrm{b}, \mathrm{A}}$ \\
\hline FZ250 & $39.71 \pm 2.43$ & $65.75 \pm 23.62$ & $53.50 \pm 26.93$ & $58.39 \pm 26.13$ & $47.99 \pm 21.34^{\mathrm{a}, \mathrm{B}}$ & $62.07 \pm 24.36^{\mathrm{a}, \mathrm{AB}}$ \\
\hline FP60 & $85.69 \pm 10.04$ & $61.42 \pm 21.96$ & $84.68 \pm 12.28$ & $84.49 \pm 18.64$ & $85.23 \pm 10.53^{\mathrm{a}, \mathrm{C}}$ & $72.19 \pm 23.06^{\mathrm{a}, \mathrm{B}}$ \\
\hline Total & & & & & $57.96 \pm 20.12^{\mathrm{a}}$ & $58.89 \pm 23.28^{\mathrm{a}}$ \\
\hline
\end{tabular}

Note: In each time point, mean values with at least a common capital letter were not statistically different (one-way ANOVA/Tukey's post hoc test). In each material, mean values of the two time point with similar lower letters were not statistically different (independent $t$-test).

For ease of illustration, comparisons of the two conditions are not reported in the table.

Table 5 Mean \pm standard deviation of Vickers microhardness values

\begin{tabular}{|l|l|l|l|l|}
\hline \multirow{2}{*}{ Material } & \multicolumn{2}{|c|}{ Coated } & \multicolumn{2}{c|}{ Uncoated } \\
\cline { 2 - 5 } & 24 hours & 60 days & 24 hours & 60 days \\
\hline Aura & $29.62 \pm 3.77^{\mathrm{a}, \mathrm{A}}$ & $30.28 \pm 5.03^{\mathrm{a}, \mathrm{A}}$ & $41.14 \pm 1.94^{\mathrm{a}, \mathrm{A}}$ & $41.94 \pm 1.85^{\mathrm{a}, \mathrm{A}}$ \\
\hline IPS & $26.13 \pm 2.08^{\mathrm{a}, \mathrm{A}}$ & $33.30 \pm 1.34^{\mathrm{b}, \mathrm{AB}}$ & $26.27 \pm 0.74^{\mathrm{a}, \mathrm{B}}$ & $33.55 \pm 1.34^{\mathrm{b}, \mathrm{B}}$ \\
\hline FZ250 & $29.70 \pm 4.93^{\mathrm{a}, \mathrm{A}}$ & $41.28 \pm 7.16^{\mathrm{b}, \mathrm{B}}$ & $44.80 \pm 3.97^{\mathrm{a}, \mathrm{A}}$ & $70.24 \pm 5.83^{\mathrm{b}, \mathrm{C}}$ \\
\hline FP60 & $50.71 \pm 4.36^{\mathrm{a}, \mathrm{B}}$ & $35.78 \pm 5.74^{\mathrm{b}, \mathrm{AB}}$ & $74.08 \pm 6.04^{\mathrm{a}, \mathrm{C}}$ & $71.75 \pm 3.32^{\mathrm{b}, \mathrm{C}}$ \\
\hline
\end{tabular}

Note: In each time point, mean values with at least a common capital letter were not statistically different (one-way ANOVA/Tukey's post hoc test). In each material, mean values of the two-time point with similar lower letters were not statistically different (independent $t$-test).

For ease of illustration, comparisons of the two conditions are not reported in the table.

At each time point and in all materials except for IPS Empress, the mean VHN of the uncoated group was significantly greater than the coated group (all $p<0.001$ ). In the case of IPS Empress, there was no significant difference between coated and uncoated conditions at any time point. In coated and uncoated IPS Empress and Z250, time 2 (60 days) provided greater mean VHN values than time 1 (24 hours; $p<0.001$ ). However, this association was inversed for coated Filtek P60 $(p<0.001)$. In other cases, no significant differences were found.

\section{Discussion}

Surface sealants and coatings have been introduced as the potential agents to improve the surface properties, ${ }^{19}$ but they tend to de-bond over time. There is little information about the long-term effects of surface resin coatings on the properties of resin composites. Therefore, the present study aimed to evaluate the effects of a nanofilled resin-base coating agent on the physical and mechanical properties of four different types of resin composites (Aura, IPS Empress direct, Filtek P60, and Filtek Z250) for up to 60 days. The null hypothesis of this study was rejected as the findings revealed that the application of G-Coat Plus altered the physical and mechanical properties of those resin composites.

\section{Water Sorption and Solubility}

Water plays an imperative role in the composite materials chemical degradation as it leads to hydrolytic reactions as well as material swelling. ${ }^{20}$ Water sorption and solubility are considered as determining factors in the restorative materials' clinical success. These factors negatively influence the resin composite's hydrolytic strength which in turn result in the staining of the material, ${ }^{21}$ degradation of its mechanical properties, ${ }^{22}$ and hydrolytic deterioration of the resin-filler bonds. ${ }^{23}$

According to the findings of the present study, there was a significant difference between the coated and uncoated specimens in terms of water sorption. In line with previous studies ${ }^{17,24}$ who showed that the application of a coating agent significantly decreased water sorption and solubility of RM-GICs, our findings verified that the coating application contributed to significantly reduced water sorption values of resin composites. Similarly, the application of a coating agent resulted in a significant reduction in the solubility values of almost all trial materials. The solubility of all of the materials showed negative values following the coating application indicating the materials' low solubility. The low solubility values of the materials after being coated might be explicated by the ability of protective coating to maintain the water balance. The application of coating protects against crack dissemination and covers the porosities, which might consequently reduce water sorption and solubility. ${ }^{24}$

Moreover, among different types of tested resin composites, the water sorption of Aura was significantly inferior to the other materials. This could be explained by the polymerization modulator chemical groups or plasticizers that might be present in the resin matrix of this bulk fill composite, reducing the polymerization shrinkage stress of the material when it is applied in bulk. ${ }^{25}$ In that case, the materials' quality 
of polymer network and their moisture resistance might be influenced by such chemical modifications. In addition, as expected, Filtek P60 showed the highest sorption values among tested materials. As previous studies have verified, the sorption values are negatively correlated to the quantity of filler loading. ${ }^{26}$ With an increase in the fillers weight \%, the polymeric matrix decreases followed by a subsequent reduction in water sorption, a phenomenon of the polymeric phase. ${ }^{27}$ Thereby, higher water sorption values of Filtek P60 could be attributed to its lower filler content.

Nevertheless, Aura and IPS Impress had the highest water solubility among the tested materials. The resin composition has an impact on the water solubility behavior of resin composites. In this regard, higher solubility values of Aura and IPS Impress could be attributed to the TEGDMA (triethylene glycol dimethacrylate) present in their matrix which is one of the smallest molecules in the tested resin composites, and can be considered as the major monomer eluted in these materials. $^{28}$

In the present study, DW was selected as the storage media in this study to simulate the liquids of oral cavity that are regularly in contact with restorations. A previous study showed that water and artificial saliva are analogous as storage media with regards to water sorption. ${ }^{27}$ The storage media was constantly substituted between each measurement to evade $\mathrm{pH}$ changes over time affecting the diffusion process and solubility. ${ }^{29}$

\section{Color Change}

Color change of dental restorations is another important characteristic of resin composites in terms of longevity. ${ }^{30}$ In the present study, coating Filtek Z250, Filtek P60, and Aura did not significantly alter their $\Delta \mathrm{E}$ values compared with the uncoated groups. However, for IPS Empress, the application of coating increased the $\Delta \mathrm{E}$ values. Therefore, the effect of coating on color stability can be considered as material dependent. One of the limitations of this study was that the effect of surface properties such as surface roughness on color stability was not evaluated alongside coating application. The authors recommend that these properties be taken into account in future studies since they act as determining factors in materials' susceptibility to staining. In consistency with our results on IPS Impress, Bagis et $\mathrm{al}^{31}$ stated that the usage of protective resin coating for resin-based restorative materials might result in more discoloration and rougher surfaces than without coating. Similarly, a previous study reported that coating applications might have negative effects on $\Delta \mathrm{E}$ of resin-based restoratives during the aging procedures. ${ }^{10}$ In another study, Halacoglu et $\mathrm{al}^{32}$ showed that applying liquid resin polish did not improve the color stability of the composite resin restorations.

In this study, significant differences in $\Delta \mathrm{E}$ values were found among materials. In both coated and uncoated groups among materials, Filtek P60 revealed the least color alteration compared with other resin composites. The setting reaction and surface reactivity are regarded as the influencing factors in dental materials' susceptibility to discoloration. ${ }^{33}$ In addition, the degree of hydrophilic/hydrophobic nature of the resin matrix might also contribute to the specimens' susceptibility to staining after being immersed in DW. Water sorption principally arises as direct absorption by the resin matrix. ${ }^{34}$ Thus, the lower $\Delta \mathrm{E}$ of Filtek P60 observed in the present study can be related to its resin matrix composition which is devoid of TEGDMA, a highly hydrophilic monomer present in other resin composites tested in this study.

The CIE L*a*b* color system, which is a three-dimensional method of color evaluation, was implemented to investigate color change in this study. $\mathrm{L}^{*}$ is the lightness factor, and $\mathrm{a}^{*} \mathrm{~b}^{*}$ are the chromocyte coordinates in the red-green axis and the yellow-blue axis. DW was used as the storage media in this test as it imitates those liquids of oral cavity that associate with composite restorations. The results of the current study revealed that all $\Delta \mathrm{E}$ values were greater than the clinically acceptable threshold of 3.3. This finding indicates that after 60 days, the color change of all of the resin composites investigated in this study were clinically visible whether coated or uncoated. ${ }^{35}$

\section{Flexural Strength}

A common method to measure the brittleness of the materials is to investigate the FS in the transverse bending. ${ }^{36}$ In brittle materials such as resin composites, microcracks present on the surface or inside the material will diminish its mechanical strength. It was expected that the surface coating agents which intend to fill surface voids and protect the surface throughout initial setting reaction, sustain adequate mechanical properties to endure forces. However, no significant differences were found between coated and uncoated materials with regard to overall mean FS values $(p=0.510)$. Our findings are in contrast with the results of Bagheri et $\mathrm{al}^{12}$ that reported an improvement in the FS of all the tested materials after applying GCP.

Among tested composites, Filtek P60 had higher FS compared with other materials in almost all experimental conditions, except for 60 days after which the coated Filtek Z250 showed greater FS. Thus, the microhybrid composites tested in this experiment performed better than the nanohybrid composites in terms of FS.

Additionally, an interaction effect was observed between materials and time. In other words, the effect of time on FS was material dependent. While Aura showed higher FS in the first 24 hours compared with 60 days, IPS revealed greater FS after 60 days.

\section{Vickers Microhardness}

Microhardness might be defined as the resistance of a material to permanent indentation or penetration. ${ }^{37}$ Any alteration in microhardness might indicate the material's setting reaction and the uncompleted maturation of the restorative material. ${ }^{38}$ In the present study, VHN were measured after 24 hours and 60 days of storage in DW. Therefore, the maturity of the resin composites was reached at the time of measurement, and any differences observed in VHN between study groups could be attributed to the effects of coating application and material composition. 
In the first 24 hours, Filtek P60 and after 60 days, Filtek Z250 and Filtek P60 showed the highest VHN in both coated and uncoated groups. The microhybrid composites exhibited higher VHN than the nanohybrid composites, whether coated or uncoated. These findings can be explicated by compositional differences between the two composites with microhybrid composites being composed of zirconia and silica filler particles that might be responsible for their greater microhardness. This finding is in agreement with previous studies which speculated that the type of inorganic filler is a potential coordinate influencing the materials' hardness, with those consisting of zirconia/silica filler particles showing higher surface hardness. ${ }^{15,39}$

The impact of water storage on the materials' microhardness is largely dependent on the type of material. As the storage time increased, so did the VHN of IPS Impress and Filtek Z250. However, for Filtek P60, a significant decrease in microhardness was observed after 60 days of water storage. While our findings found no deleterious effect of 2-month water aging on the microhardness of Filtek Z250, Moraes et al ${ }^{40}$ reported a significant softening effect of 6-month water storage on the surfaces of this resin composite.

Furthermore, the application of a coating agent led to a significant reduction in the microhardness values as the uncoated materials revealed higher VHN than the coated composites both after 24 hours and 60 days of water immersion. This finding was expected as the resin-enriched top layer is a much weaker phase than the bulk of the cured material and its presence would probably result in greater indenter's depth of penetration of to the subsurface restorative material. Similarly, Bertrand et a ${ }^{19}$ revealed that the application of resin coating caused a decrease of the microhardness of the resin composite's surface. Bagheri et a ${ }^{12,15}$ also reported a significantly lower VHN in their study following the application of GCP compared with the uncoated groups.

These findings demonstrate that each restorative dental material entails its own treatment modality to obtain surfaces, which have least color change along with the highest strength. In addition, Filtek Z250 and Filtek P60 with the highest VHN and FS values can be ideally used in posterior region while IPS Impress and Aura should be used with caution in the load-bearing area. Although coating resin composites might be beneficial in terms of reducing water sorption and solubility, the application of resin coating might be a risk factor for $\triangle \mathrm{E}$, VHN, and FS in the long run. Although the effect of water aging was taken into account in this study, further in vivo experiments are required to substantiate the data obtained in this research. The effect of resin coating on the surface roughness of resin composites would be an interesting topic for future studies.

\section{Conclusion}

Within the limitations of the present study, it was concluded that coating the resin composites might be beneficial in terms of reducing water sorption and solubility, protective resin coating for resin composites might not be an advantage for the materials' color stability, Vickers microhardness, and
FS in the long term. Among tested composites, Filtek P60 exhibited the least color change and the highest water sorption, Vickers microhardness, and FS.

\section{Funding \\ None.}

\section{Conflict of Interest}

None declared.

\section{Acknowledgments}

Authors are specially grateful to Ivoclar Vivadent, GC, 3M; and SDI Victoria, Australia for generously providing the materials. The authors also thank Dr. M. Vosoughi for his contribution and advice in the statistical analyzes.

\section{References}

1 Fahl Júnior $\mathrm{N}$. The aesthetic composite anterior single crown restoration. Pract Periodontics Aesthet Dent 1997;9(1):59-70, quiz 72

2 Mount G, Hume W, Knowledge Books and Software. Dentil: Varsity Lakes, Queensland, Australia; 2005

3 Roulet J-F. Benefits and disadvantages of tooth-coloured alternatives to amalgam. J Dent 1997;25(6):459-473

4 Chadwick RG, McCabe JF, Walls AW, Storer R. The effect of storage media upon the surface microhardness and abrasion resistance of three composites. Dent Mater 1990;6(2):123-128

5 Bagheri R, Tyas MJ, Burrow MF. Comparison of the effect of storage media on hardness and shear punch strength of tooth-colored restorative materials. Am J Dent 2007;20(5):329-334

6 Meşe A, Burrow MF, Tyas MJ. Sorption and solubility of luting cements in different solutions. Dent Mater J 2008;27(5):702-709

7 Ellakuria J, Triana R, Mínguez N, et al. Effect of one-year water storage on the surface microhardness of resin-modified versus conventional glass-ionomer cements. Dent Mater 2003;19(4):286-290

8 Munack J, Haubert H, Dogan S, Geurtsen W. Effects of various storage media on surface hardness and structure of four polyacid-modified composite resins ("compomers") Clin Oral Investig 2001;5(4):254-259

9 Buchalla W, Attin T, Hilgers R-D, Hellwig E. The effect of water storage and light exposure on the color and translucency of a hybrid and a microfilled composite. J Prosthet Dent 2002;87(3):264-270

10 Zimmerli B, Koch T, Flury S, Lussi A. The influence of toothbrushing and coffee staining on different composite surface coatings. Clin Oral Investig 2012;16(2):469-479

11 Topcu FT, Sahinkesen G, Yamanel K, Erdemir U, Oktay EA, Ersahan S. Influence of different drinks on the colour stability of dental resin composites. Eur J Dent 2009;3(1):50-56

12 Bagheri R, Taha NA, Azar MR, Burrow MF. Effect of G-Coat Plus on the mechanical properties of glass-ionomer cements. Aust Dent J 2013;58(4):448-453

13 VG S. To evaluate the effect of surface coating on three different types glass ionomer restorations. Biomed Pharmacol J 2015;8:445-449

14 Hepdeniz OK, Temel UB, Ugurlu M, Koskan O. The effect of surface sealants with different filler content on microleakage of Class V resin composite restorations. Eur J Dent 2016;10(2):163-169

15 Bagheri R, Palamara J, Mese A, Manton DJ. Effect of a self-adhesive coating on the load-bearing capacity of tooth-coloured restorative materials. Aust Dent J 2017;62(1):71-78

16 Hankins AD, Hatch RH, Benson JH, Blen BJ, Tantbirojn D, Versluis A. The effect of a nanofilled resin-based coating on 
water absorption by teeth restored with glass ionomer. J Am Dent Assoc 2014;145(4):363-370

17 Jafarpour D, Mese A, Ferooz M, Bagheri R. The effects of nanofilled resin-based coatings on the physical properties of glass ionomer cement restorative materials. J Dent 2019;89:103177

18 Bonifácio CC, Werner A, Kleverlaan CJ. Coating glass-ionomer cements with a nanofilled resin. Acta Odontol Scand 2012;70(6):471-477

19 Bertrand MF, Leforestier E, Muller M, Lupi-Pégurier L, Bolla M. Effect of surface penetrating sealant on surface texture and microhardness of composite resins. J Biomed Mater Res 2000;53(6):658-663

20 Geurtsen W. Substances released from dental resin composites and glass ionomer cements. Eur J Oral Sci 1998;106(2 Pt 2) :687-695

21 Um CM, Ruyter IE. Staining of resin-based veneering materials with coffee and tea. Quintessence Int 1991;22(5):377-386

22 Yiu CK, King NM, Pashley DH, et al. Effect of resin hydrophilicity and water storage on resin strength. Biomaterials 2004;25(26):5789-5796

23 Ferracane JL. Hygroscopic and hydrolytic effects in dental polymer networks. Dent Mater 2006;22(3):211-222

24 Jevnikar P, Serša I, Sepe A, Jarh O, Funduk N. Effect of surface coating on water migration into resin-modified glass ionomer cements: a magnetic resonance micro-imaging study. Magn Reson Med 2000;44(5):686-691

25 Goracci C, Cadenaro M, Fontanive L, et al. Polymerization efficiency and flexural strength of low-stress restorative composites. Dent Mater 2014;30(6):688-694

26 Mortier E, Gerdolle DA, Jacquot B, Panighi MM. Importance of water sorption and solubility studies for couple bonding agentresin-based filling material. Oper Dent 2004;29(6):669-676

27 Alshali RZ, Salim NA, Satterthwaite JD, Silikas N. Longterm sorption and solubility of bulk-fill and conventional resin-composites in water and artificial saliva. J Dent 2015;43(12):1511-1518

28 Örtengren U, Wellendorf H, Karlsson S, Ruyter IE. Water sorption and solubility of dental composites and identification of monomers released in an aqueous environment. J Oral Rehabil 2001;28(12):1106-1115

29 Örtengren U, Langer S, Göransson A, Lundgren T. Influence of $\mathrm{pH}$ and time on organic substance release from a model dental composite: a fluorescence spectrophotometry and gas chromatography/mass spectrometry analysis. Eur J Oral Sci 2004;112(6):530-537

30 Mundim FM, Pires-de-Souza FdeC, Garcia LdaF, Consani S. Colour stability, opacity and cross-link density of composites submitted to accelerated artificial aging. Eur J Prosthodont Restor Dent 2010;18(2):89-93

31 Bagis B, Tüzüner T, Turgut S, Korkmaz FM, Baygın Ö, BağıŞ YH. Effects of protective resin coating on the surface roughness and color stability of resin-based restorative materials. Sci World J 2014;2014:832947

32 Halacoglu DM, Yamanel K, Basaran S, Tuncer D, Celik C. Effects of staining and bleaching on a nanohybrid composite with or without surface sealant. Eur J Dent 2016;10(3):361-365

33 Abu-Bakr N, Han L, Okamoto A, Iwaku M. Color stability of compomer after immersion in various media. J Esthet Dent 2000;12(5):258-263

34 Oysaed H, Ruyter IE. Water sorption and filler characteristics of composites for use in posterior teeth. J Dent Res 1986;65(11):1315-1318

35 Magni E, Zhang L, Hickel R, Bossù M, Polimeni A, Ferrari M. SEM and microleakage evaluation of the marginal integrity of two types of class $\mathrm{V}$ restorations with or without the use of a light-curable coating material and of polishing. J Dent 2008;36(11):885-891

36 Miyazaki M, Moore BK, Onose H. Effect of surface coatings on flexural properties of glass ionomers. Eur J Oral Sci 1996;104(5-6):600-604

37 O'Brien W, Physical Properties in Dental Materials and Their Selection. Illinois: Quintessence Publishing Co, 1997

38 Bourke AM, Walls AW, McCabe JF. Light-activated glass polyalkenoate (ionomer) cements: the setting reaction. J Dent 1992;20(2):115-120

39 Taha N, Ghanim A, Tavangar M. Comparison of mechanical properties of resin composites with resin modified glass ionomers. J Dent Biomater 2015;2:47-53

40 de Moraes RR, Marimon JLM, Schneider LF, Sinhoreti MAC, Correr-Sobrinho L, Bueno M. Effects of 6 months of aging in water on hardness and surface roughness of two microhybrid dental composites. J Prosthodont 2008;17(4):323-326 